Scientific Paper

\title{
Dosimetric proprieties of FBX dosimeter for high energy photon and electron beams
}

\author{
Ouiza Moussous ${ }^{1, a}$, Toufik MEDJADJ ${ }^{1}$, Saad KHOUDRI ${ }^{2}$ \\ ${ }^{I}$ Department of medical physics, Nuclear Research Center of Algiers (NRCA), 02 Boulevard Frantz Fanon B.P. 399 Alger- \\ Gare, Algeria \\ ${ }^{2}$ Centre Anti Cancèreux, Blida, Algeria \\ ${ }^{a}$ E-mail address: o.moussous@crna.dz.
}

(received 9 February 2017; revised 31 May 2017; accepted 5 July 2017)

\begin{abstract}
The aim of this work is to characterize the ferrous sulfate-benzoic acid-xylenol orange (FBX) aqueous chemical dosimeter developed at our laboratory, prepared using ultra pure water, by measuring the absorption spectrum, dose response curve, precision and accuracy, energy and dose rate dependency and stability of response. The FBX readings were evaluated by using an accurate spectrophotometer. Experimental data were obtained using various nominal energies $6 \mathrm{MV}, 18 \mathrm{MV}, 12 \mathrm{MeV}$, and $15 \mathrm{MeV}$, including the ${ }^{60} \mathrm{Co} \gamma$-rays beam. The calibration of the dosimeters was performed using the ionization chamber as a reference dosimeter. The results show that the FBX dosimeter has a good precision of about $0.2 \%$, no significant energy, dose rate dependence and a linear dose-response relationship in the $1-5$ Gy range.
\end{abstract}

Key words: FBX dosimeter; dosimetric proprieties; spectrophotometry; high energy beams.

\section{Introduction}

High energy photon and electron beams are widely used in radiation therapy. The quantity of interest is the absorbed dose determined in a water phantom. Accurate determination of absorbed radiation energy is important for applications of ionising radiation in medicine. The FBX aqueous chemical dosimetry system that was developed for low level dose measurements by Gupta and co-workers [1-3] has found many applications in radiotherapy owing to its improved sensitivity at low doses as compared to Fricke system. It has been found useful for depth dose measurements, output calibration [4] quality assurance in external beam therapy [5], brachytherapy source calibration [6], in vivo dose measurements [7], and for measurement of virtual wedge profiles [8]. In this system absorbed doses are measured in terms of the oxidation yield of ferric ions produced by irradiation. The ferric ions form a color complex with xylenol orange which is measured spectrophotometrically at $548 \mathrm{~nm}$.

Additional FBX dosimetric properties obtained with high energy photon and electron beams using a reading instrument of high performance are presented in this work. A basic dosimeter response curve was plotted for ${ }^{60} \mathrm{Co} \gamma$-rays and then a comparison was made with high energy x-rays and electron. A precision and accuracy obtained are also reported. The measurements were carried out in the secondary standard dosimetry laboratory at NRCA Algiers.

\section{Materials and Methods}

\section{Preparation of solution}

All glassware was thoroughly rinsed with high purity water and dried in an oven. The FBX dosimeter solution contained, 0.2 $\mathrm{mol} / \mathrm{L}$ of ferrous ammonium sulfate $\left(\mathrm{Fe}\left(\mathrm{NH}_{4}\right)_{2}\left(\mathrm{SO}_{4}\right)_{2} \times 6 \mathrm{H}_{2} \mathrm{O}\right)$ (analytical reagent grade from Fluka), $5 \mathrm{~mol} / \mathrm{L}$ of benzoic acid (analytical reagent grade from Fluka), and $0.20 \mathrm{~mol} / \mathrm{L}$ tetra sodium salt of xylenol orange (XO) in $40.0 \mathrm{~mol} / \mathrm{L}$ concentrated sulphuric acid $\left(\mathrm{H}_{2} \mathrm{~S}_{4}\right)$ (analytical reagent grade from Merck). Ferrous ammonium sulphate, benzoic acid and xylenol orange were weighed using a precision and analytical balance model KERN ARS Version 2.0 (Germany). The solution was prepared using ultra pure water $(18.2 \mathrm{M} \Omega \mathrm{cm})$ provided by a arium ${ }^{\circledR} 611$ Ultrapure water system (Sartorius, Germany). The solution was freshly prepared, air saturated, stabilized for about an hour and used within 1 day.

\section{Method of irradiation}

The proprieties of FBX dosimeter were studied using high energy photon and electron beams of nominal energies $6 \mathrm{MV}$, $18 \mathrm{MV}$ X-rays and $12 \mathrm{MeV}, 15 \mathrm{MeV}$ electrons, including the ${ }^{60} \mathrm{Co} \gamma$-ray. The irradiation units used are the Varian linear accelerators, Clinac $1800 \mathrm{C}$ for X-rays, Clinac $2100 \mathrm{C}$ for electrons and an Eldorado 78 unit for the ${ }^{60} \mathrm{Co}$.

All the beams were calibrated in a water phantom using a PTW UNIDOS 10002 electrometer with $0.6 \mathrm{~cm}^{3}$ (2175) 
Farmer-type ion chamber and Roos 34001 parallel plate chamber of volume $0.35 \mathrm{~cm}^{3}$. The Roos ionization chamber is inherently waterproof. The Farmer-type is used with the 1-mm thick PMMA waterproofing sleeve. All measurements were done by strictly adhering to the standards conditions stipulated in the IAEA TRS 398 dosimetry protocol [9].

In photon beams all irradiations were performed in horizontal beam geometry using a cubic water phantom (IAEA standard phantom) of side $30 \mathrm{~cm}$, having a $10 \mathrm{~cm} \times 10 \mathrm{~cm}$ insert with a $1 \mathrm{~mm}$ thick PMMA window. The depth measurement was 5 $\mathrm{g} / \mathrm{cm}^{2}$ for ${ }^{60} \mathrm{Co}$ and $6 \mathrm{MV}, 10 \mathrm{~g} / \mathrm{cm}^{2}$ for $18 \mathrm{MV}$. The absorbed dose rate in the ${ }^{60} \mathrm{Co}$ beam was approximately $0.5 \mathrm{~Gy} / \mathrm{min}$ on the measurement date at the reference point, $1.8 \mathrm{~Gy} / \mathrm{min}$ and $2.5 \mathrm{~Gy} / \mathrm{min}$ at $6 \mathrm{MV}$ and $18 \mathrm{MV}$ respectively. In electron beams the irradiations were carried out in vertical beam geometry using a PTW ID water phantom of side $40 \mathrm{~cm}$. In the $12 \mathrm{MeV}$ electrons beam the depth measurement was $2.7 \mathrm{~g} / \mathrm{cm}^{2}$. At $15 \mathrm{MeV}$ measurements were made at $3.9 \mathrm{~g} / \mathrm{cm}^{2}$ depth. The absorbed dose rate was approximately $2.5 \mathrm{~Gy} / \mathrm{min}$ at $12 \mathrm{MeV}$, $5 \mathrm{~Gy} / \mathrm{min}$ at $15 \mathrm{MeV}$. The fixed square field size was set to 10 $\times 10 \mathrm{~cm}^{2}$ at the source-surface distance (SSD) equaled to 80 $\mathrm{cm}$ and $100 \mathrm{~cm}$ for ${ }^{60} \mathrm{Co}$ and linear accelerator, respectively.

Usually, the irradiation was done after one day from the preparation of FBX solution. The dosimeter ampoules used for irradiation had the following dimensions: inner diameter 10.6 $\mathrm{mm}$, height $31 \mathrm{~mm}$ (referred to the top surface of the liquid in the ampoule) and Pyrex wall thickness $0.5 \mathrm{~mm}$. The ampoules were first sealed with the paraffin stopper and then positioned with their geometrical center at the measurement point in the water phantom using a suitable PMMA stand.

\section{Dose, energy and dose rate dependence}

As a first step, FBX dosimeters were exposed to a photon beam from a ${ }^{60} \mathrm{Co}$-source in order to study the dosimeter responses to the absorbed dose and also to establish the dosimeter calibration. The samples were irradiated at doses ranging from 1 to $30 \mathrm{~Gy}$ and, then, analyzed. Eleven dose levels were selected in the range investigated. To evaluate the reproducibility of the dosimeter, at each dose level five FBX dosimeters were irradiated in the same conditions.

The energy dependence of FBX dosimeter, was evaluated for a dose of $5 \mathrm{~Gy}$ by means of the calibration coefficient $\mathrm{N}_{\mathrm{w}}=$ $\mathrm{D}_{\mathrm{w}} / \Delta \mathrm{OD}$, where $\mathrm{D}_{\mathrm{w}}$ is the value of absorbed dose in water as measured by ionization chamber, and $\Delta \mathrm{OD}$ is the difference in optical density between the irradiated and unirradiated FBX solution.

To check the dose rate dependence, the FBX samples were irradiated with absorbed dose of $5 \mathrm{~Gy}$, in different radiation sources, ${ }^{60} \mathrm{Co}$ gamma radiation, high energy photons and electron beams. The dose rate range was 0.6-5 Gy/min.

\section{Spectrophotometric analysis}

The optical density readings of FBX solution were carried out by a Varian Cary 100 UV-VIS double beam spectrophotometer
(Varian, Australia). The absorbance readings were made at a wavelength of $548 \mathrm{~nm}$ with a spectral bandwidth of $1.5 \mathrm{~nm}$, using quartz micro cells having a path length of $10 \mathrm{~mm}$ and an optical window $4 \mathrm{~mm}$ wide and $45 \mathrm{~mm}$ high.

\section{Results and discussion}

The present work refers to the following proprieties of FBX dosimeters exposed to high energy photon and electron beams: precision and accuracy, energy dependence, dose rate dependence and linearity.

\section{Absorption spectrum}

Figure 1, illustrates the optical absorption spectra for unirradiated, irradiated FBX dosimeter solution by using ${ }^{60} \mathrm{Co}$ $\gamma$-rays with dose $5 \mathrm{~Gy}$ and the spectrum of optical density change. The difference spectrum shows a very broad maximum centered at about $540 \mathrm{~nm}$. However since in the sensitive range of interest spectra lie around $548 \mathrm{~nm}$, this value was considered for further analysis of the experimental data.

The molar extinction coefficient, was measured in this study and found to be $14740 \pm 50 \mathrm{M}^{-1} \mathrm{~cm}^{-1}$ at $548 \mathrm{~nm}$. The error given represents the standard deviation of the mean of three independent sets of measurements carried out.

\section{Precision and Accuracy}

Table 1, shows the results of the reproducibility check carried out with FBX solution prepared freshly on one day. The standard deviation of the mean is found to be less than $0.3 \%$ for photons and electrons. Not significant differences were observed between the standard deviation according to the dose for the same beam. This observation is identical for all the used beams. For a considered beam, the average of all the standard deviation is equal to $0.3 \%$ and characterizes the average reproducibility of the dosimeter for the measure of dose.

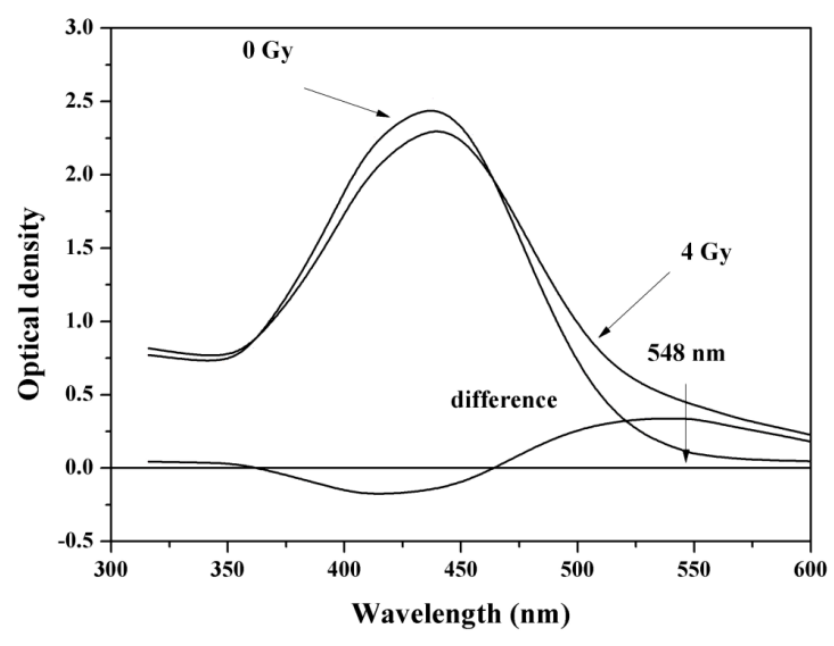

Figure 1. The change in the absorption spectrum of an irradiated ferrous sulphate benzoic acid xylenol orange (FBX) dosimeter. The 0 Gy and 4 Gy spectra were measured with air as the reference. The difference spectrum has a broad peak centered at about $540 \mathrm{~nm}$. 
Table 1. Reproducibility check carried out with the FBX dosimeter.

\begin{tabular}{|c|c|c|c|c|c|c|c|c|c|c|}
\hline \multirow{2}{*}{$\begin{array}{l}\text { Dose } \\
(G y)\end{array}$} & \multicolumn{2}{|c|}{${ }^{60} \mathrm{Co}$} & \multicolumn{2}{|c|}{$6 \mathrm{MV}$} & \multicolumn{2}{|c|}{$18 \mathrm{MV}$} & \multicolumn{2}{|c|}{$12 \mathrm{MeV}$} & \multicolumn{2}{|c|}{$15 \mathrm{MeV}$} \\
\hline & $\overline{\Delta \mathbf{O D}}^{\text {(a) }}$ & $\mathrm{s}(\%)^{(\mathrm{b})}$ & $\overline{\Delta \mathbf{O D}}^{\text {(a) }}$ & $\mathrm{s}(\%)^{(\mathrm{b})}$ & $\overline{\Delta \mathbf{O D}}^{(\text {a) }}$ & $\mathrm{s}(\%)^{(\mathrm{b})}$ & $\overline{\Delta \mathbf{O D}}^{\text {(a) }}$ & $\mathrm{s}(\%)^{(\mathrm{b})}$ & $\overline{\Delta \mathbf{O D}}^{\text {(a) }}$ & $\mathrm{s}(\%)^{(\mathrm{b})}$ \\
\hline 1 & 0.0839 & 0.20 & 0.0821 & 0.30 & 0.0840 & 0.30 & 0.0842 & 0.30 & 0.0845 & 0.30 \\
\hline 2 & 0.1678 & 0.20 & 0.1665 & 0.20 & 0.1678 & 0.20 & 0.1720 & 0.21 & 0.1730 & 0.21 \\
\hline 3 & 0.2499 & 0.30 & 0.2500 & 0.30 & 0.2600 & 0.20 & 0.2450 & 0.30 & 0.2460 & 0.30 \\
\hline 4 & 0.3329 & 0.20 & 0.3334 & 0.23 & 0.3329 & 0.20 & 0.3290 & 0.23 & 0.3300 & 0.23 \\
\hline 5 & 0.4154 & 0.20 & 0.4120 & 0.20 & 0.4154 & 0.30 & 0.4150 & 0.32 & 0.4164 & 0.32 \\
\hline
\end{tabular}

(a) Represents the mean value of five readings of $\Delta \mathrm{OD}$.

(b) Experimental standard deviation of $\triangle \mathrm{OD}$ obtained using five dosimeters irradiated at the same dose.

Table 2. Accuracy check carried out with the FBX dosimeter.

\begin{tabular}{|c|c|c|c|c|c|c|}
\hline \multirow{2}{*}{$\begin{array}{l}\text { Delivered } \\
\text { dose (Gy) }\end{array}$} & \multicolumn{3}{|c|}{ Measured dose (Gy) } & \multicolumn{3}{|c|}{ Difference (\%) } \\
\hline & ${ }^{60} \mathrm{Co}$ & 18 MV X-rays & $12 \mathrm{MeV}$ electr. & ${ }^{60} \mathrm{Co}$ & 18 MV X-rays & $12 \mathrm{MeV}$ electr. \\
\hline 1 & 1.002 & 1.006 & 1.003 & -0.2 & 0.6 & 0.3 \\
\hline 2 & 2.013 & 2.005 & 2.070 & 0.6 & 0.2 & 3.5 \\
\hline 3 & 3.002 & 3.064 & 2.957 & 0.1 & 2.0 & 1.4 \\
\hline 4 & 4.002 & 3.981 & 3.978 & 0.1 & -0.5 & 0.5 \\
\hline 5 & 4.990 & 4.990 & 5.016 & 0.2 & 0.2 & 0.3 \\
\hline
\end{tabular}

This result shows that the precision of FBX dosimeters is not significantly dependent on radiation quality.

The long term reproducibility of the FBX dosimeter was evaluated using the ${ }^{60}$ Co therapy unit over 350 days. Figure 2 shows the relative response of FBX dosimeter normalized to the mean as a function of days elapsed since the first measurement. After correction for source decay assuming the half-life of ${ }^{60} \mathrm{Co}$ radioactivity to be 1925 days, the FBX dosimeter shows random differences of $0.2 \%$ as indicated in Figure 2.

The accuracy of the FBX dosimeter was checked by calculating the difference between the dose delivered and that determined using the linear regression equation applied to the calibration curve Figure 3. The results obtained are presented in Table 2.

\section{Dose response}

In relation to the dose-response curve Figure 3, it was observed a linear behavior in the range $1 \mathrm{~Gy}-5 \mathrm{~Gy}$, and, probably, a saturation effect after 5 Gy due to chemical changes and reagent consumption. The regression analysis showed that the differential absorbance sensitivity, (slope), was $0.0832 \mathrm{~Gy}^{-1}$. The correlation coefficient $\mathrm{R}^{2}$ was 0.9996 which showed an excellent linear fit for the dose range of interest. The linear interval tested is consistent with the results cited in the literature $[4,11]$ and is adequate for radiotherapy purposes. The radiation chemical yield $G\left(\mathrm{Fe}^{+3}\right)$ value, which is a product of this slope and the molar extinction coefficient, $\varepsilon$, was evaluated and found to be $55.96( \pm 0.40) \times 10^{-7} \mathrm{~mol} \mathrm{~J}{ }^{-1}$. The error given is the combined uncertainty $(1 \sigma)$ calculated according to the International Organization for Standardization Guide of uncertainties in Table 3 [10]. The $G$ value obtained in the present investigation is comparable within experimental errors with previously reported value $[11,12]$.

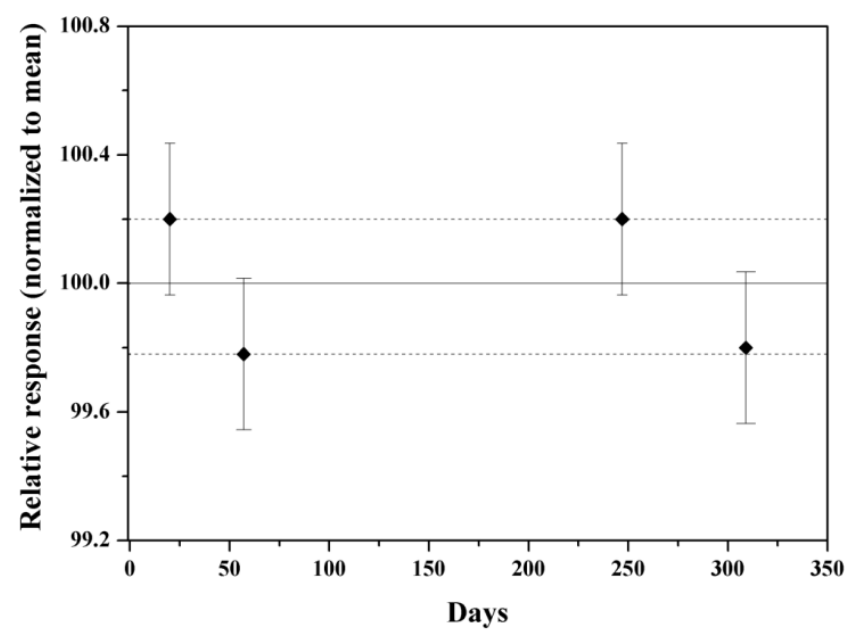

Figure 2. Long term reproducibility of the FBX dosimeter. The error bars are the standard errors of the plotted measured values.

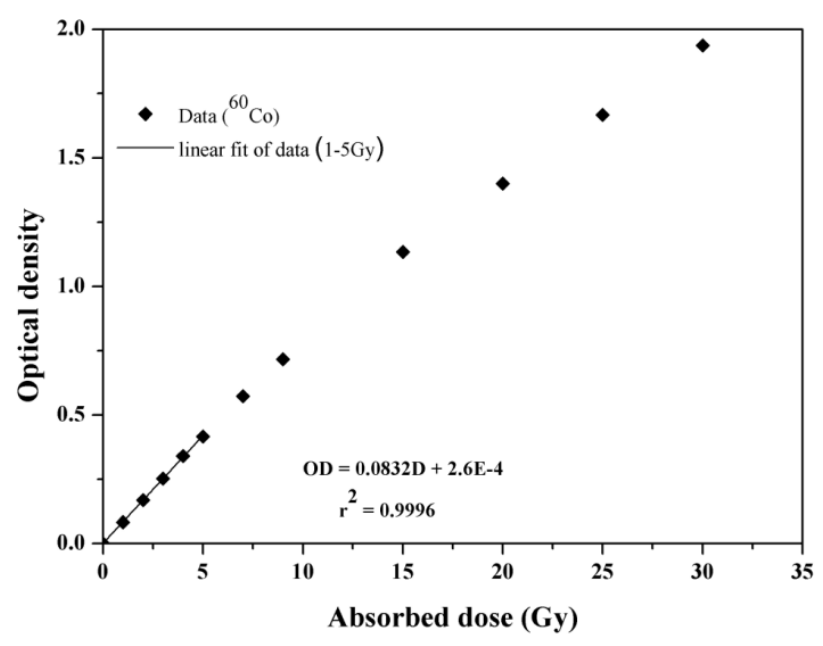

Figure 3. FBX dosimeter dose response curve for ${ }^{60} \mathrm{Co}$ energy photons, $10 \times 10 \mathrm{~cm}^{2}, 80 \mathrm{~cm}$ SSD at $5 \mathrm{~cm}$ depth in water. The relative standard deviation of the mean is $0.3 \%$. 
Table 3. Uncertainty budget for the $G\left(\mathrm{Fe}^{3+}\right)$ value obtained at ${ }^{60} \mathrm{Co}$ beam.

\begin{tabular}{lcc}
\hline \hline Source of uncertainties & Type A (\%) & Type B (\%) \\
\hline $\mathbf{1}$ Factors influencing the reference $\boldsymbol{D}_{\mathrm{w}}$ & & \\
$\mathrm{N}_{\mathrm{D}, \mathrm{w}}$ calibration coefficient reported by IAEA & & 0.49 \\
Dosimeter reading & 0.01 & \\
Constancy of the ionization chamber & & 0.10 \\
Temperature: diff. between $\mathrm{T}^{\circ}$ inside cavity & & 0.06 \\
Thermometer resolution & & 0.02 \\
Pressure & 0.20 & 0.06 \\
$\mathbf{2}$ Factors influencing FBX solution & & 0.30 \\
$\Delta$ OD measurement & & 0.10 \\
$\varepsilon$ measurement & & 0.17 \\
$\rho$ & & 0.62 \\
\hline Q & & \\
\hline Relative combined standard uncertainty & 0.65 \\
\hline \hline
\end{tabular}

\section{Energy dependence}

Figure 4 is a plot of the $N_{w}$ relative values as a function of photon and electron energy in the range $1.2515 \mathrm{MeV}$ for a dose of $5 \mathrm{~Gy}$. The uncertainty of the experimental points was estimated to be $0.7 \%$ for ${ }^{60} \mathrm{Co}$ and $1.7 \%$ for high energy photon and electron beams respectively obtained from the combination of the overall uncertainty of FBX dosimeter and ionization chamber. The solid line represents the average of all the data. The dashed lines represent values which are $1 \%$ higher and $1 \%$ lower than the average. The dosimeters showed random differences less of $1 \%$ energy dependence over the range of nominal photon and electron energies examined in this study.

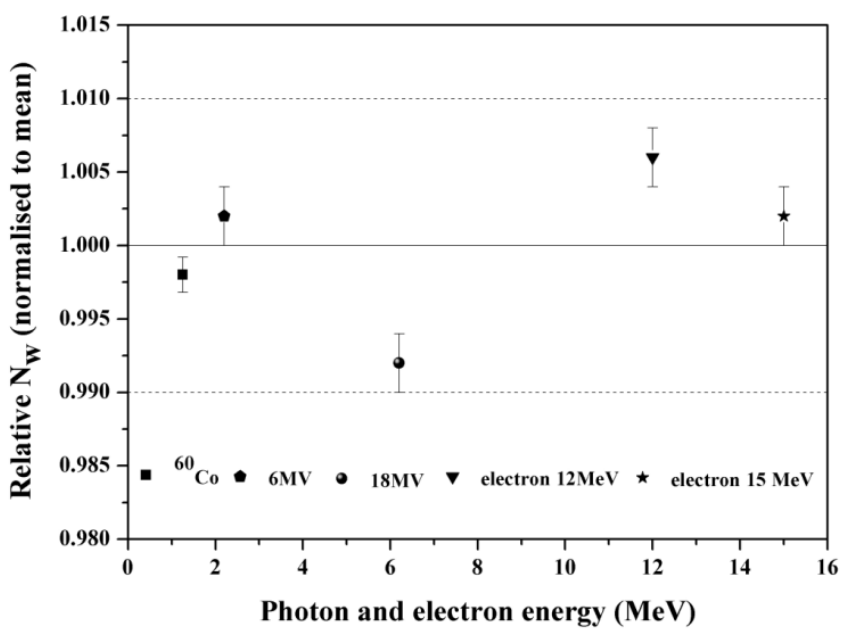

Figure 4. Relative calibration factors of FBX dosimeter for photon and electron beams. The error bars correspond to a $68 \%$ confidence level. The solid line represents the average of all data. The $x$-ray energies published in units of accelerating potential were converted to approximate equivalent photon energies using data from BJR Supplement 25 [12,13], e.g. $6 \mathrm{MV}$ became $2.2 \mathrm{MeV}$ and $18 \mathrm{MV}$ became $6.2 \mathrm{MeV}$.

\section{Dose rate dependence}

Figure 5 shows the relative response of FBX dosimeter normalized to the mean as a function of dose rate. Little doserate effects less than $1 \%$ deviation within experimental error were observed. The data suggest that there is no measurable effect within the dose rate interval studied. This fact agrees well with results reported elsewhere [4].

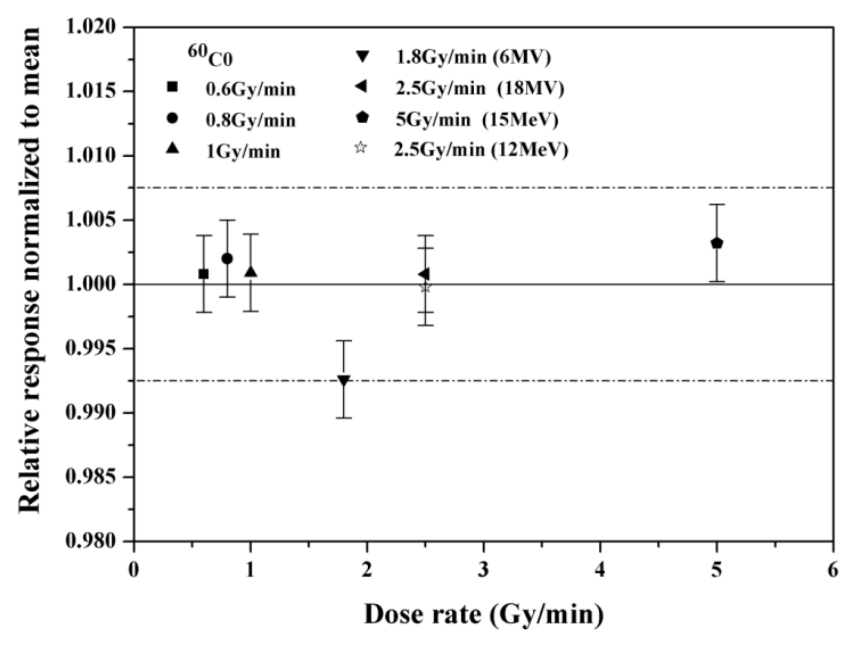

Figure 5. Relative response to the mean value of the FBX dosimeter as a function of dose rate. An error bar shows the standard deviation $(1 \sigma)$ of each set of measurements. The solid line represents the average of all data.

\section{Conclusion}

In this work the basic proprieties of the FBX dosimetry system prepared using ultra pure water were investigated. An efficient spectrophotometer for optical density evaluation was used. The dosimeter demonstrated a reproducible linear dose response up to $5 \mathrm{~Gy}$, which is suitable for many therapeutic applications. The linear interval tested is consistent with the results cited in the literature [4,11]. No significant (less than $1 \%$ ) dose rate or energy dependence for this dosimeter was observed over the range studied. Previous studies have shown an effect of $2 \%$ [15]. The radiation chemical yield $G\left(\mathrm{Fe}^{+3}\right)$ of FBX dosimeter was found to be $55.96( \pm 0.40) \times 10^{-7} \mathrm{~mol} \mathrm{~J}^{-1}$, which is consistent with the observations of $[11,12]$. The results tabulated in Table 1 showed that reproducibility of the FBX measurements is less than $0.3 \%$. It was found to be better than early data reported elsewhere (more than $1 \%$ ) $[4,15]$. The results showed that long term reproducibility is $0.2 \%$. It was found to be similar with data reported for standard Fricke dosimeter in the literature [16]. The results in this study support the conclusion that the FBX is a good practical clinical dosimeter.

\section{Acknowledgments}

Grateful acknowledgement is made to Dr. B. Hocini (Mustapha Hospital, Algiers) for allowing us to use their hospital's equipment. We also thank radio-physicists of their staff for their help in making the measurements. 


\section{References}

[1] Gupta BL. Low-level dosimetric studies with the FeSO4-benzoic acid-xylenol orange system. In: Dosimetry in agriculture, industry, biology and medicine. Proceedings of a symposium organized by the IAEA [International Atomic Energy Agency] 17021 April 1972; Vienna, Austria. Vienna: IAEA; 1973: 4210432.

[2] Gupta BL, Gomathy KR. Consistency of ferrous sulfate-benzoic acid-xylenol orange dosimeter. Int J Appl Radiat Isot. 1974;25(1112):509-513.

[3] Gupta BL, Bhat RM, Gomathy KR Suseela B. Radiation chemistry of the ferrous sulphate-benzoic acid-xylenol orange dosimeter. Radiat Res. 1978;75(2):269-277.

[4] Gupta BL, Kini UR, Bhat RM, Madhvanath U. Use of the FBX dosimeter for the calibration of cobalt-60 and high energy teletherapy machines. Phys Med Biol. 1982;27(2):235-245.

[5] Gupta BL, Narayan GR, Bhat RM, Kini UR, et al. Use of the FBX dosimeter for the quality assurance of ${ }^{60} \mathrm{Co}$ and high-energy teletherapy machines by mail. Phys Med Biol. 1992;37(11):2095-2102.

[6] Madhvanath U, Kini UR, Gupta BL. Calibration of brachytherapy sources using ferrous sulfate-benzoic acid-xylenol orange dosimeter. Int J Appl Radiat Isot. 1976;27(8):443-446.

[7] Semwal MK, Bansal AK, Thakur PK, Vidyasagar PB. In-vivo (entrance) dose measurements in external beam radiotherapy with aqueous FBX dosimetry system. J Med Phys. 2005;30(1):32-35.

[8] Semwal MK, Bansal AK, Thakur PK, Vidyasagar PB. FBX aqueous chemical dosimeter for measurement of virtual wedge profiles. $\mathbf{J}$ Appl Clin Med Phys. 2008;9(2):206-210.

[9] IAEA. Absorbed dose determination in external beam radiotherapy: an international code of practice for dosimetry based on standards of absorbed dose to water. Vienna: International Atomic Energy Agency, Technical Reports Series TRS-398; 2000.

[10] ISO. Guide to the Expression of Uncertainty in Measurement: International Organization for Standardization, ISO 1993.

[11] Bhat AA, Choudhary D, Sarma A, et al. Response of an FBX dosimeter to high $\mathrm{LET}{ }^{7} \mathrm{Li}$ and ${ }^{12} \mathrm{C}$ ions. Radiat Phys Chem. 2003;68(5):909-916.

[12] Gupta BL, Nilekani SR. Ferrous ion oxidation by $\mathrm{H}, \mathrm{OH}$ and $\mathrm{H}_{2} \mathrm{O}_{2}$ in aerated FBX dosimetry system. Radiat. Phys. Chem. 1998;53(6):643-650.

[13] Smith CW. Orthovoltage x-ray beams (0.5 mm-4.0 mm Cu HVL). Br J Radiol Suppl. 1996;25:24-38.

[14] Jordan TJ. Megavoltage x-ray beams: 2-50 MV. Br J Radiol Suppl. 1996;25:62-109.

[15] Brindha S, Rose JV, Sathyan S, et al. Modified ferrous ammonium sulfate benzoic acid xyelenol orange (MFBX) and thermoluminescent dosimeters - a comparative study. Phys Med Biol. 2002;47(11):N153-N158.

[16] Klassen NV, Shortt KR, Seuntjens J, Ross CK. Fricke dosimetry: the difference between G(Fe3+) for 60Co-rays and high-energy xrays. Phys Med Biol. 1999 44(7): 1609-1624. 